\title{
Tumor Findings Original Result
}

National Cancer Institute

\section{Source}

National Cancer Institute. Tumor Findings Original Result. NCI Thesaurus. Code C119934.

The outcome of the tumor findings assessment as orig inally received or collected. 